\title{
Association of Leptin with Body Pain in Women
}

\author{
Jarred Younger, $\mathrm{PhD},{ }^{1}$ Kristopher Kapphahn, $\mathrm{MS}^{2}$, Kathleen Brennan, MD, \\ Shannon D. Sullivan, MD, PhD, and Marcia L. Stefanick, $\mathrm{PhD}^{5}$
}

\begin{abstract}
Leptin, an appetite-regulatory hormone, is also known to act as a proinflammatory adipokine. One of the effects of increased systemic leptin concentrations may be greater sensitivity to pain. We report the results of two studies examining the association between leptin and pain: a small pilot longitudinal study, followed by a large cross-sectional study. In Study 1, three women with physician-diagnosed fibromyalgia provided blood draws daily for 25 consecutive days, as well as daily self-reported musculoskeletal pain. Daily fluctuations in serum leptin were positively associated with pain across all three participants $(F(1,63)=12.8, p<0.001)$, with leptin predicting $\sim 49 \%$ of the pain variance. In Study 2, the relationship between leptin and body pain was examined in a retrospective cross-sectional analysis of 5676 generally healthy postmenopausal women from the Women's Health Initiative. Leptin levels obtained from single blood draws were tested for a relationship with selfreported body pain. Body mass index (BMI) was also included as a predictor of pain. Both leptin and BMI were found to be independently associated with self-reported pain ( $p=0.001$ and $p<0.001$, respectively), with higher leptin levels and greater BMI each being associated with greater pain. Leptin appears to be a predictor of body pain both within- and between-individuals and may be a driver of generalized pain states such as fibromyalgia.
\end{abstract}

\section{Introduction}

$\mathbf{I}$ $\mathrm{N}$ THESE TWO STUDIES, we explore the relationship between leptin and generalized musculoskeletal pain. Leptin is a protein produced primarily by white adipose tissue. Originally recognized as an appetite-regulatory hormone, leptin has more recently been demonstrated to also act as a proinflammatory adipokine. ${ }^{1,2}$ Leptin administration in humans has been shown to elevate many clinical markers of systemic inflammation, including C-reactive protein. $^{3}$

Leptin-mediated inflammation is associated with increased pain sensitivity. A small number of animal studies have shown that intrathecally or peripherally injected leptin can induce systemic hyperalgesia and allodynia. ${ }^{4-6}$ While no human pain studies have involved the experimental administration of leptin, there are several reports that leptin levels in synovial fluid and in peripheral blood are positively associated with pain severity in individuals with osteoarthritis. ${ }^{7-10}$ Serum leptin may also be increased in the widespread chronic pain condition fibromyalgia, ${ }^{11,12}$ although not all studies have supported a leptin-fibromyalgia link. ${ }^{13}$

We have previously reported that day-to-day fluctuations in serum leptin predict daily fatigue severity in women with chronic fatigue syndrome. ${ }^{14}$ On days when leptin concentrations are higher, women self-reported greater fatigue severity. Out of 50 cytokines and chemokines examined in that previous study, leptin was the analyte that was most consistently associated with symptom severity.

Many women with chronic fatigue syndrome also meet diagnostic criteria for fibromyalgia. ${ }^{15}$ While not necessarily suggesting overlapping etiologies, the characterizing symptoms are similar between the two conditions. ${ }^{16}$ Fibromyalgia is a chronic pain condition of uncertain etiology that is characterized by widespread musculoskeletal pain and sensitivity to mechanical pressure in soft tissue. Sufferers also often report profound fatigue, disruption in cognitive processes, sleep difficulties, and exacerbation of symptoms following physical or mental exertion. Because of the potential

\footnotetext{
${ }^{1}$ University of Alabama at Birmingham, Birmingham, Alabama.

${ }^{2}$ Stanford University, Stanford, California.

${ }^{3}$ University of California, Los Angeles, California.

${ }^{4}$ Georgetown University, Washington, DC.

${ }^{5}$ Department of Medicine, Stanford Prevention Research Center, Stanford University, Stanford, California.
}

(C) Jarred Younger, et al. 2016; Published by Mary Ann Liebert, Inc. This Open Access article is distributed under the terms of the Creative Commons Attribution Noncommercial License (http://creativecommons.org/licenses/by-nc/4.0/), which permits any noncommercial use, distribution, and reproduction in any medium, provided the original author(s) and the source are credited. 
overlap between chronic fatigue syndrome and fibromyalgia, we sought to explore the role that leptin may also play in widespread musculoskeletal pain.

In this report, we explore the results of two studies that examined the relationship between leptin and widespread body pain. Study 1 was a small proof-of-concept analysis of three women with fibromyalgia. The participants provided blood samples and self-reported body pain severity scores for 25 consecutive days. We hypothesized that daily serum leptin would be positively correlated with self-reported pain. While leptin was our primary predictor, we were also more broadly interested in the role of inflammatory processes in driving chronic pain. We therefore examined 50 other cytokines and chemokines in an exploratory analysis, to identify potential predictors of pain for further study.

In Study 2, we performed a cross-sectional analysis between leptin and general body pain in over 5000 women from the Women's Health Initiative (WHI) Observational Study who had previously provided blood samples and ratings of body pain. ${ }^{17}$ Participants also completed clinical measures of body mass index (BMI). Because BMI is positively correlated with leptin ${ }^{18}$ and associated with self-reported pain ${ }^{19-24}$ and fibromyalgia, ${ }^{25,26}$ we controlled for BMI in our analyses. This existing dataset therefore provided the opportunity to conduct the first large-scale cross-sectional analysis of the relationships between leptin and body pain. Our general hypothesis was that both higher serum leptin concentrations and greater BMI would be independently associated with selfreported general pain. Our specific hypotheses, registered in advance with the WHI Publications Committee, were as follows:

Hypothesis 1: Secreted leptin is positively associated with greater self-reported pain.

Hypothesis 2: BMI is positively associated with pain.

Hypothesis 3: Leptin is positively associated with pain after controlling for BMI.

Hypothesis 4: BMI is positively associated with pain after controlling for leptin.

Because Study 1 and Study 2 used substantially different study designs and analytic approaches, they are covered separately in this article. Methods and Results for Study 1 are presented and then Methods and Results for Study 2. Those sections are followed by a General Discussion section.

\section{Study 1: Pilot Longitudinal Analysis of Daily Leptin and Pain in Three Women with Fibromyalgia}

\section{Methods: Study 1}

Participants. Participants in this unfunded pilot study were three Caucasian women, aged 31, 35, and 40, who met both the American College of Rheumatology 1991 and 2010 diagnostic criteria for fibromyalgia. Participants were not part of any previous analyses. Because of the expense of daily immune monitoring, only three individuals were recruited and enrolled. No participants had a history of autoimmune or rheumatologic disorder. All participants had normal levels of erythrocyte sedimentation rate, C-reactive protein, thyroid hormone levels, rheumatoid factor, and antinuclear antibodies. All participants reported an average daily pain level of at least 60 out of 100 (pain visual analog scale) and none were taking prescription medications.
Protocol. Participation for each individual lasted for 33 days. Screening and consenting were performed in the Adult and Pediatric Pain Laboratory at Stanford University. All procedures were approved by the Institutional Review Board at Stanford University.

After screening and consenting, participants submitted a blood sample to screen for exclusionary autoimmune and inflammatory conditions. Participants were then given an Android-based smartphone for reporting daily pain severity. Participants provided ratings of general body pain severity with a single-item measure, "Please rate your general level of body pain" on a 0-100 scale. "0" was anchored as "no pain" and "100" as "worst pain imaginable." Participants completed the measure before going to sleep and were instructed to provide a report that reflected their overall experience over the entire day. The survey also contained 17 other items that examined symptoms such as fatigue, headaches, and sleep quality. None of those items were examined in this analysis.

Pain severity reports were provided for the duration of the study participation (32 days). In the first week (7 days), pain reports were collected as a baseline and habituation period. No blood draws were taken in the first week. The week baseline also allowed the study team to ensure that participants would reliably submit their symptom reports.

Following the 7-day baseline period, participants started the 25-day blood draw phase of the study. During the blood draw phase, participants came to the Clinical Translational Research Unit (CTRU) at Stanford University every morning. The time of day was locked to control for any possible diurnal fluctuations in leptin. Venipuncture was performed using a 23-gauge needle in the antecubital fossa and $\sim 8 \mathrm{cc}$ of blood was collected in serum separating tubes. The draw site was rotated to reduce discomfort from the daily repeated venipuncture. After remaining at room temperature for 30 minutes, the serum layer was spun off into eight cryovials and stored at $-80^{\circ} \mathrm{C}$. Participants continued to provide symptom severity reports every morning and night during the blood draw phase of the study.

Leptin and cytokine quantification. All assays were performed by the Human Immune Monitoring Center (HIMC) at Stanford University. A human 51-plex Luminex cytokine kit was purchased from Affymetrix and used according to manufacturer's recommendations. More detailed procedures have been provided previously. ${ }^{14}$ All samples were batch processed on a single plate.

\section{Statistical analysis}

The 51 cytokines (see full analyte list in Table 1) were tested for associations with pain via linear mixed models in SPSS v21 (IBM). Linear mixed models allow for proper analysis when intensive longitudinal data are nested within subjects. Each blood analyte was tested with a separate model. The dependent variable was self-reported daily pain. The participant identification number was entered as the nested within-subjects identifier and study day (day 1-25) as the repeated-measures index. Lagged relationships were not explored in the models. Blood analyte (e.g., leptin) was entered as a fixed, continuous covariate predictor. All variables were participant centered to avoid influence of between-subject 
Table 1. Full List of Analytes Assessed in Study 1

\begin{tabular}{lcclcc}
\hline Analyte & $\mathrm{F}$ & $\mathrm{p}$ & Analyte & $\mathrm{F}$ & $\mathrm{p}$ \\
\hline Leptin & $\mathbf{1 2 . 8 0 4}$ & $\mathbf{0 . 0 0 0 7}$ & IL-4 & 1.351 & 0.25 \\
IL-17F & 8.016 & 0.006 & IL-5 & 1.353 & 0.25 \\
Eotaxin & 7.587 & 0.008 & IL-6 & 1.089 & 0.301 \\
VEGF & 6.93 & 0.011 & IL-7 & 1.022 & 0.317 \\
V-CAM1 & 6.723 & 0.012 & IL-8 & 0.975 & 0.327 \\
IFN- $\beta$ & 6.134 & 0.016 & IP10 & 0.787 & 0.379 \\
CD40Ligand & 5.617 & 0.021 & LIF & 0.73 & 0.397 \\
ENA78 & 4.917 & 0.031 & M-CSF & 0.664 & 0.418 \\
FGFb & 4.433 & 0.04 & MCP-1 & 0.646 & 0.425 \\
G-CSF & 3.997 & 0.05 & MCP-3 & 0.622 & 0.433 \\
GM-CSF & 3.953 & 0.051 & MIG & 0.545 & 0.463 \\
GRO- $\alpha$ & 3.929 & 0.052 & MIP-1 $\beta$ & 0.474 & 0.494 \\
HGF & 3.929 & 0.052 & MIP-1 $\alpha$ & 0.427 & 0.516 \\
ICAM-1 & 3.724 & 0.058 & NGF & 0.266 & 0.608 \\
IFN- $\alpha$ & 3.019 & 0.087 & PAI-1 & 0.234 & 0.63 \\
IFN- $\gamma$ & 2.742 & 0.103 & PDGFBB & 0.209 & 0.649 \\
IL-10 & 2.484 & 0.12 & RANTES & 0.052 & 0.821 \\
IL-12 p70 & 2.488 & 0.121 & Resistin & 0.05 & 0.823 \\
IL-12 p40 & 2.435 & 0.124 & SCF & 0.034 & 0.855 \\
IL-13 & 2.419 & 0.125 & sFAS_lig & 0.018 & 0.892 \\
IL-15 & 2.201 & 0.143 & TGF- $\alpha$ & 0.009 & 0.926 \\
IL-17 & 2.212 & 0.144 & TGF- $\beta$ & 0.001 & 0.988 \\
IL-1 $\alpha$ & 1.963 & 0.166 & TNF- $\alpha$ & 0.001 & 0.995 \\
IL-1 $\beta$ & 1.89 & 0.174 & TNF- $\beta$ & $($ below detection) \\
IL-1Ra & 1.673 & 0.203 & Trail & (below detection) \\
IL-2 & 1.353 & 0.249 & VCAM-1 & (below detection) \\
\hline
\end{tabular}

The significance threshold was Bonferroni adjusted to 0.001 (significant results are marked in bold font). Analytes are listed in descending order of statistical significance as a predictor of selfreported pain.

IFN, interferon; IL, interleukin; MCP, monocyte chemoattractant protein; MIP, macrophage inflammatory protein; TGF, transforming growth factor; TNF, tumor necrosis factor.

differences on model results. An autoregressive covariance structure (AR1) was used for nested data and a restricted maximum likelihood used for testing of fixed effects.

Because of the large number of cytokines being assessed separately, a Bonferroni adjustment was made to the statistical threshold so that false positives would be properly controlled. A Bonferroni adjustment with an alpha of 0.05 and 51 tests yielded an adjusted threshold of $p<0.001-$ controlling the chance of any false positives at $5 \%$.

\section{Results: Study 1}

Participant 1 missed one blood draw session. Participant 3 missed two days of self-report pain measures. Days with missing data were excluded from analyses. Across all three participants, there were 72 usable days of data (96\% successful completion rate).

Body weight for the three participants was as follows: $60.2,82.1$, and $101.2 \mathrm{~kg}$. Leptin concentrations for the three participants were as follows: $0.7 \mathrm{ng} / \mathrm{mL}$ (range $0.2-2.0$ ), $10.2 \mathrm{ng} / \mathrm{mL}$ (range $5.8-17.1$ ), and $20.5 \mathrm{ng} / \mathrm{mL}$ (11.21-38.7). Mean leptin values were typical for healthy women with the observed body weights.

Linear mixed models revealed that daily leptin levels were significantly associated with daily pain reports $(F(1,63)=$ $12.804, p=0.0007)$. On days with greater self-reported pain, leptin levels were higher. Sequence plots and scatterplots are provided for all participants in Figure 1. No other cytokine predictors survived the statistical threshold. Statistical results for all cytokines are provided in Table 1.

\section{Study 2: Cross-Sectional Analysis of Leptin and Body Pain in 6000 Postmenopausal Women}

\section{Methods: Study 2}

Participants. The analytic cohort was a subsample of postmenopausal women, aged 50-79, who were enrolled in the large WHI Observational Study between 1993 and 1998. Detailed descriptions of the recruitment and screening methods and data collection have been provided previously. ${ }^{17}$ Briefly, 93,726 postmenopausal women representing a diverse ethnic background and socioeconomic status range completed a comprehensive battery of health measures at baseline visits. A subset of participants also provided blood samples that were subsequently assayed for leptin quantification. Of 6,286 participants who had at least one serum leptin measurement, 123 were excluded from these analyses for missing data on BMI or self-reported pain. An additional 472 women were excluded because of a diagnosed autoimmune or rheumatologic disease (rheumatoid arthritis, multiple sclerosis, lupus, and amyotrophic lateral sclerosis) or because of self-reported opioid analgesic use. The final analytic cohort consisted of 5676 individuals.
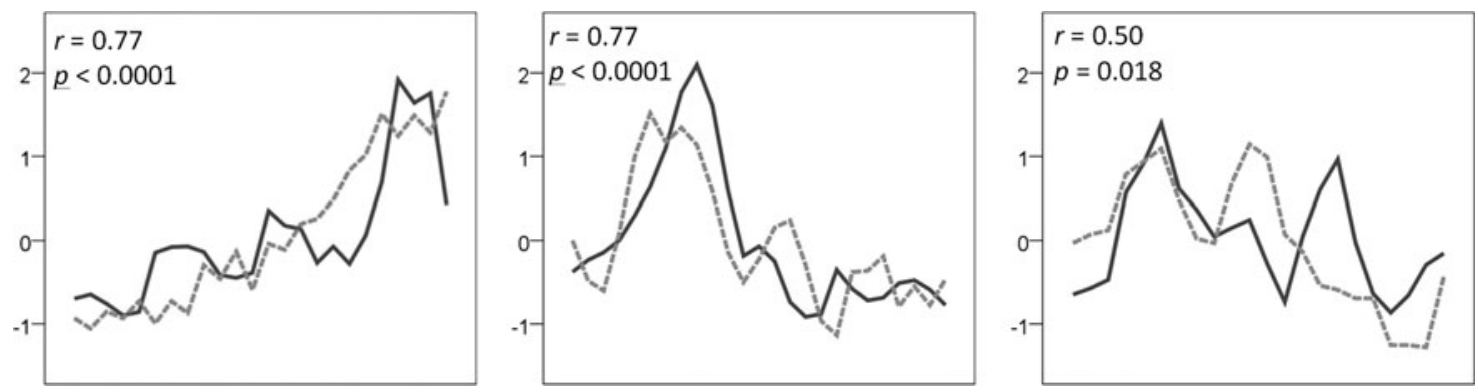

FIG. 1. Relationship between daily serum leptin and daily pain in three women with fibromyalgia. Sequence plots. Solid line represents leptin and dashed line represents pain over 25 consecutive days ( $x$-axis). Both leptin and pain have been individual centered with a $z$-score transformation to put all data on the same $y$-scale. For general descriptive purposes, individual Pearson's correlations are provided. The relationship between leptin and pain for the three individuals was as follows: $0.77(p<0.001), 0.77(p<0.001)$, and $0.50(p=0.018)$. 
Protocol. Body pain at study entry was assessed using a single-item question. Subjects were asked "During the past four weeks, how much bodily pain have you had?" Responses were provided on an ordinal scale, with the following options: none, very mild, mild, moderate, and severe. For modeling purposes, answers were coded as integers ranging from 1 to 5 with 1 corresponding to "none" and 5 with "severe." Pain from other sources (e.g., illness or injuries) was assessed with other questions that were not examined in this analysis.

Height and weight were collected by trained study staff. Weight was measured to the nearest $0.1 \mathrm{~kg}$ and height measured to the nearest $0.1 \mathrm{~cm}$. BMI was calculated using the standard formula of weight $(\mathrm{kg})$ divided by the square of height $\left(\mathrm{m}^{2}\right)$. Participants were fasting at the time of the blood draw, which was taken between 7 and $11 \mathrm{am}$. There was an average of $23.8(\mathrm{SD}=79.9)$ days between the blood draw and pain measurement. Venous blood samples were collected into serum separating (SST; $N=55,1.0 \%$ ), ethylenediaminetetraacetic acid (EDTA; $N=1712,30.2 \%$ ), or citrate $(N=3909,68.9 \%)$ tubes. Samples were spun down and the serum layer was extracted and stored at $-80^{\circ} \mathrm{C}$.

Leptin quantification. Three separate research groups later analyzed the stored serum samples for concentrations of leptin. Leptin concentrations for EDTA plasma and citrate samples were established using the multiplex assay (Milliplex Human Adipokine Panel B; Millipore). Leptin assays demonstrated an interassay coefficient of variation (CV) of 9\%. Additional information about processing techniques has been published previously. ${ }^{27}$ Leptin levels of serum from SSTs were determined with radioimmunoassay using commercial kits (Lunco Research, Inc.). The interassay CV for leptin was 5.5\%. Six subjects with SST assays had multiple assays; for these subjects, within-subject average leptin data were used. Eighty-five subjects had multiple leptin measurements using more than one assay type. For those individuals, only the most common (i.e., citrate) assay type was retained for analysis.

\section{Statistical analysis}

The sole dependent variable for all models was self-reported body pain. Two predictor (regressor) variables were used in the models. The primary regressor was serum leptin level. The second regressor was BMI. BMI range groups were created for under/normal weight (BMI $0-25 \mathrm{~kg} / \mathrm{m}^{2}$ ), overweight (BMI $\left.25-29.925 \mathrm{~kg} / \mathrm{m}^{2}\right)$, and obese $\left(\right.$ BMI $>30 \mathrm{~kg} / \mathrm{m}^{2}$ ).

All models were tested using parametric linear regression and adjusted for race and age. All leptin values were logtransformed. Because leptin concentration levels vary by the collection tube type (SST, EDTA, and citrate), all models were controlled for tube type. $p$-Values $<0.05$ were considered statistically significant, and exact $p$-values were reported down to the $p=0.001$ level of significance. Three models were tested:

In Model 1 (testing Hypothesis 1), body pain was the dependent variable; independent variables were as follows: leptin level, specimen type, age, and race.

In Model 2 (testing Hypothesis 2), body pain was the dependent variable; independent variables were as follows: $\mathrm{BMI}$, age, and race.
In Model 3 (testing Hypotheses 3 and 4), body pain was the dependent variable; independent variables were as follows: leptin, BMI, a leptin-BMI interaction term, specimen type, age, and race.

\section{Results: Study 2}

Baseline characteristics of the analytic cohort and median leptin values are presented in Table 2. Median leptin $(\mathrm{ng} / \mathrm{mL})$ values were: 13.8 (no pain), 15.3 (very mild pain), 18.3 (mild pain), 20.3 (moderate pain), and 23.0 (severe pain). A Pearson's $r$ showed that the two predictors (leptin and continuous BMI) were correlated at 0.64 .

Statistics for all models are presented in Table 3. In Model 1 , serum leptin was tested as a predictor of self-reported body pain. The relationship was significant $(p<0.001)$. Higher reports of pain were associated with greater leptin concentrations; a twofold increase in leptin was associated with a mean increase of 0.17 (95\% CI 0.14-0.20) points on the $0-4$ pain scale (Table 3 ).

In Model 2, we found a significant positive correlation between BMI and self-reported body pain $(p<0.001)$. Higher reports of pain were associated with greater BMI, with overweight subjects having an average pain score of 0.30 (95\% CI $0.21-0.38$ ) points greater than those in the underweight/normal group $(p<0.001)$. Obese subjects had an average pain score that was 0.69 (95\% CI $0.59-0.78$ ) points greater than those in the underweight/normal group $(p<0.001)$.

In Model 3, the leptin $\times$ BMI interaction was not significantly associated with self-reported pain $(p=0.38)$. Both leptin and BMI remained significant predictors of pain when controlling for the other factor $(p=0.001$ and $p<0.001$, respectively). Every $100 \%$ increase in logged leptin levels was associated with a mean increase of 0.07 (95\% CI 0.04-0.11) points on the 0-4 pain scale after adjusting for BMI. Because interpreting logged values of leptin is not intuitive, we also present the model results using raw leptin values. Translating to raw values, every $\mathrm{ng} / \mathrm{mL}$ increase of leptin was associated with a $0.4 \%$ increase of pain from the sample mean pain of 1.4. The slope would predict a $74 \%$ increase of pain when moving from the minimum to maximum observed leptin values in our sample.

Holding leptin constant, subjects in the overweight BMI group had mean pain scores that were 0.20 (95\% CI 0.110.30 ) points greater than those in the underweight/normal group, and obese subjects had mean pain scores that were 0.51 (95\% CI 0.39-0.63) points greater than those in the underweight/normal group.

\section{General Discussion}

We observed that leptin is associated with self-reported body pain both longitudinally within-person and betweenpersons. In Study 1, day-to-day fluctuations in serum leptin covaried with daily fluctuations of pain. In Study 2, leptin levels were positively correlated with pain, even after controlling for BMI. Our results agree with previous animal and human studies that suggest leptin may heighten pain sensitivity, perhaps via proinflammatory activity. Leptin has been associated with a large number of inflammatory diseases such as rheumatoid arthritis, lupus, and multiple sclerosis. ${ }^{28}$ 
Table 2. Baseline Characteristics and Leptin Concentrations by Pain Level in Study 2

\begin{tabular}{|c|c|c|c|c|c|}
\hline & None & Very Mild & Mild & Moderate & Severe \\
\hline$N$ & 1344 & 2120 & 1216 & 1012 & 49 \\
\hline Leptin (ng/mL) & 13.8 & 15.3 & 18.3 & 20.3 & 23.0 \\
\hline IQR & 16.7 & 18.4 & 22.9 & 23.3 & 29.6 \\
\hline BMI, kg/m² (SD) & $25.28(5.71)$ & $26(6.39)$ & $27.29(7.36)$ & $27.94(7.73)$ & $28.92(10.27)$ \\
\hline \multicolumn{6}{|l|}{ BMI category $(\%)$} \\
\hline$<25 \mathrm{~kg} / \mathrm{m}^{2}$ & 46.8 & 40.8 & 31.8 & 27.4 & 20.7 \\
\hline $25-29.9 \mathrm{~kg} / \mathrm{m}^{2}$ & 33.6 & 34.9 & 36.2 & 33.3 & 36.8 \\
\hline$\geq 30 \mathrm{~kg} / \mathrm{m}^{2}$ & 17.9 & 23.1 & 30.7 & 38.5 & 41.4 \\
\hline \multicolumn{6}{|l|}{ Age group $(\%)$} \\
\hline $50-54$ & 8.9 & 8.6 & 6.9 & 7.8 & 6.9 \\
\hline $55-59$ & 12.3 & 13.7 & 11.3 & 12.5 & 12.6 \\
\hline $60-69$ & 45.8 & 44.1 & 45.2 & 39.5 & 44.3 \\
\hline $70-79$ & 33.0 & 33.6 & 36.5 & 40.3 & 36.2 \\
\hline \multicolumn{6}{|l|}{ Race/Ethnicity } \\
\hline White & 69.2 & 74.7 & 76.9 & 73.8 & 64.4 \\
\hline Black & 10.7 & 10.1 & 8.5 & 12.1 & 20.1 \\
\hline Hispanic & 6.9 & 5.7 & 6.6 & 6.6 & 8.0 \\
\hline Asian/PI & 10.1 & 5.8 & 5.0 & 3.5 & 4.6 \\
\hline Native American/Alaskan & 2.6 & 2.8 & 1.8 & 2.9 & 1.1 \\
\hline Other & 0.3 & 0.7 & 0.9 & 0.7 & 0.6 \\
\hline
\end{tabular}

Pain was rated on a 0 (none) to 4 (severe) scale. Leptin is presented as median and IQR because of nonnormal distribution (wide and right skewed).

BMI, body mass index; IQR, interquartile range.

Several mechanisms for leptin-induced inflammation have been proposed. Proposed critical links include signal transducer and activator of transcription 3 (STAT3), ${ }^{29}$ IL-6, and IL-18. ${ }^{30}$ Leptin provocation of macrophages can lead to increased output of pronociceptive factors that increase pain processing. ${ }^{31}$ Leptin has also been observed to prime microglia, leading to exaggerated IL-1beta production in response to stimuli, ${ }^{32}$ and triggering microglial production of IL-6. ${ }^{33}$ Abnormal microglial activity is currently being closely examined as an important driver of chronic pain states, ${ }^{34}$

Table 3. Associations Between Leptin, BMi, and Self-Reported Pain in Study 2

\begin{tabular}{|c|c|c|c|c|c|c|c|}
\hline Model & Design & $\begin{array}{l}\text { Leptin } \\
\text { aggregation } \\
\text { scheme }\end{array}$ & $\mathrm{N}$ & $\begin{array}{l}\text { Dependent } \\
\text { variable }\end{array}$ & $\begin{array}{l}\text { Independent } \\
\text { variable }\end{array}$ & Estimate $(95 \% \mathrm{CI})$ & $\mathrm{p}$ \\
\hline 1 & $\begin{array}{l}\text { Association between pain } \\
\text { and } \log (\text { leptin) }\end{array}$ & $\begin{array}{l}\text { Averaged } \\
\text { within subject }\end{array}$ & 5676 & Pain & Leptin $\mathrm{ng} / \mathrm{mL}^{\mathrm{a}}$ & $0.17(0.14,0.20)$ & $<0.001$ \\
\hline 2 & $\begin{array}{l}\text { Unadjusted association } \\
\text { between pain and BMI }\end{array}$ & NA & 5676 & Pain & BMI & & $<0.001$ \\
\hline 2 & $\begin{array}{l}\text { Unadjusted association } \\
\text { between pain and BMI }\end{array}$ & NA & 5676 & Pain & $\begin{array}{l}\text { BMI: } 25-30 \\
\text { versus } 0-25\end{array}$ & $0.30(0.21,0.38)$ & \\
\hline 2 & $\begin{array}{l}\text { Unadjusted association } \\
\text { between pain and BMI }\end{array}$ & NA & 5676 & Pain & $\begin{array}{l}\text { BMI: } 30-70 \\
\text { versus } 0-25\end{array}$ & $0.69(0.59,0.78)$ & \\
\hline 3 & $\begin{array}{l}\text { Association between BMI } \\
\text { and pain, adjusted for } \\
\text { leptin levels }\end{array}$ & $\begin{array}{l}\text { Averaged } \\
\text { within subject }\end{array}$ & 5676 & Pain & BMI & & $<0.001$ \\
\hline 3 & $\begin{array}{l}\text { Association between BMI } \\
\text { and pain, adjusted for } \\
\text { leptin levels }\end{array}$ & $\begin{array}{l}\text { Averaged } \\
\text { within subject }\end{array}$ & 5676 & Pain & $\begin{array}{l}\text { BMI: } 25-30 \\
\text { versus } 0-25\end{array}$ & $0.20(0.11,0.30)$ & \\
\hline 3 & $\begin{array}{l}\text { Association between BMI } \\
\text { and pain, adjusted for } \\
\text { leptin levels }\end{array}$ & $\begin{array}{l}\text { Averaged } \\
\quad \text { within subject }\end{array}$ & 5676 & Pain & $\begin{array}{l}\text { BMI: } 30-70 \\
\text { versus } 0-25\end{array}$ & $0.51(0.39,0.63)$ & \\
\hline 3 & $\begin{array}{l}\text { Association between leptin } \\
\text { and pain, adjusted for } \\
\text { BMI levels }\end{array}$ & $\begin{array}{l}\text { Averaged } \\
\text { within subject }\end{array}$ & 5676 & Pain & Leptin $\mathrm{ng} / \mathrm{mL}^{\mathrm{a}}$ & $0.07(0.04,0.11)$ & 0.001 \\
\hline
\end{tabular}

Model 1 tests leptin as the sole predictor. Model 2 tests BMI as the sole predictor. Model 3 enters both leptin and BMI simultaneously. Pain was rated on a 0 (none) to 4 (severe) scale. All models were adjusted for sample type (citrate, ethylenediaminetetraacetic acid, or serum-separating tube), age, and race/ethnicity.

${ }^{\mathrm{a}}$ Variable was $\log _{2}$ transformed before fitting. Estimate corresponds to the expected change in pain for a twofold increase in leptin (ng/mL). 
and signalers such as leptin that modulate the microglial activity may be important targets for future pain therapies. Leptin may also regulate other aspects of pain processing, such as decreasing descending pain modulatory signals via the rostral ventromedial medulla. ${ }^{35}$

Increasingly, obesity is being viewed as a proinflammatory state with several adverse consequences. ${ }^{36}$ Leptin is one mechanism that may link obesity to inflammation and the associated physical and psychological diseases that are observed more often in obese individuals. ${ }^{37-40}$ Leptin levels, however, are also controlled by factors not associated with BMI, such as sleep ${ }^{41}$ and acute infection. ${ }^{42}$

Further information regarding the link between leptin and pain may help drive novel treatments for chronic pain. There are a number of behavioral techniques for lowering leptin levels, including calorie restriction and weight reduction, ${ }^{43}$ as well as physical exercise. ${ }^{44}$ Leptin levels may also be modulated pharmacologically via novel suppressing and antagonist agents ${ }^{45,46}$ and modulation of naturally occurring hormones such as ghrelin. ${ }^{47}$ Recent evidence has suggested that administration of leptin antagonists can prevent and reverse injury-induced neuropathic pain in animal models. ${ }^{48}$ With leptin-modulating pharmaceuticals, however, care would need to be taken to avoid adversely impacting appetite, caloric intake, and reproductive function.

Neither one of our studies were designed to determine causality. While leptin is available for human use as injectable metreleptin, its significant side-effect profile makes it impossible to obtain for research use at this time. We note that animal research with leptin administration support a causal influence of leptin on pain. ${ }^{4,6}$ Furthermore, limited human data suggest that current leptin levels may predict future pain severity. ${ }^{49}$

While leptin was associated with pain severity both withinand between-individuals, the strength of the effect was most significant in the within-person analyses, predicting $49 \%$ of the pain variance. The leptin-pain link in the cross-sectional study, although statistically significant, was weak and would not likely have clinical utility as a marker for pain in the general population. We are not aware of other blood-based markers of general pain being reported in a highly heterogeneous and largely healthy sample, and such markers are typically more useful as indicators of specific pain conditions. Our analyses were performed on a convenience sample in which the pain measures and blood samples were collected on separate days. Leptin can vary significantly within individuals even within days, ${ }^{50,51}$ so the study did not have the optimal timing of data collection to ensure a sensitive test of the hypothesis. In Study 1, we observed the relationship between pain and leptin to drop to zero with a 4-day lag. Future studies examining pain and leptin should aim to take the measurements as close to each other as possible.

The large study also used a crude measure of pain severity with only five possible levels of pain. More advanced modeling approaches, such as structural equation modeling, may also be useful in determining the relative contribution of body weight and leptin to pain severity, as well describing important mediating and moderating effects. High correlations between predictors can adversely affect the performance of linear regressions, although we observed the Pearson's correlation between BMI (continuous) and leptin to be a reasonable 0.64 . Nonetheless, it is important to note that BMI is a significant driver of leptin and it is difficult conceptually to separate the two. Future studies should also use more accurate methods for body composition, including those that can quantify adipose tissue. Despite those limitations, we found that pain levels linearly increased with higher leptin levels, as predicted.

It should also be highlighted that Study 1 used individuals with a diagnosed pain condition, while Study 2 excluded individuals with autoimmune disorders such as rheumatoid arthritis (although individuals reporting "severe", pain may be suspected of having an undiagnosed condition). Unfortunately, the WHI database does not reliably capture fibromyalgia diagnoses, so we were not able to determine if leptin levels are elevated in those individuals. It is likely that the sample contained some individuals with a diagnosis of fibromyalgia. Future analyses should examine leptin levels in individuals with well-characterized chronic pain diagnoses. Because we have also identified a link between leptin and fatigue, ${ }^{14}$ future research may also need to examine multiple clinical outcomes to understand the full implications of fluctuating leptin levels.

We note that, while the majority of available literature points to a positive association between leptin and pain, there are a few studies that report leptin may actually reduce the experience of pain $^{52}$ and serve a neuroprotective function. ${ }^{53,54}$ Also, at least one study reported that lower levels of leptin are associated with greater pain severity in fibromyalgia patients. ${ }^{55}$ Given the large day-to-day changes in leptin concentrations we observed (even when holding time of day constant), care may need to be taken when interpreting results of cross-sectional studies. It is possible that a single leptin assessment is not adequate for determining an individual's average leptin concentration.

The mean leptin values we observed in Study 1 were all within expected ranges for the given body weights, but there are no clinical standards for determining elevated leptin levels for a given individual. It is not likely that a leptin assay would have clinical utility as a single or infrequent screening. Leptin may work best as a longitudinal variable, although it is unknown if it can represent a biomarker for fibromyalgia disease course over time. ${ }^{13}$ The relationship between leptin and pain may be modulated by other factors, with estrogen being one possibility. ${ }^{56}$ Also, current concepts such as leptin insensitivity $^{57}$ will need to be carefully considered when examining leptin and pain. Future studies should examine factors known to interact with leptin or its signaling pathways, such as ghrelin, sex hormones, insulin, and neuropeptide $\mathrm{Y}$.

While not meeting the Bonferroni threshold, we identified other analytes potentially associated with self-reported pain, including IL-17F and eotaxin. While discussing the potential role these analytes may play in pain is outside the scope of this report, we do note that previous studies have identified both IL-17 and eotaxin to be elevated in fibromyalgia patients. ${ }^{58,59}$ These analytes, and others, may deserve more attention in future studies. However, we caution that these findings are exploratory, not involving a priori hypotheses, and from a very small sample of individuals.

In summary, we add to a growing body of literature that suggests a role for leptin in acute and chronic pain. As secreted leptin levels are substantially higher in women than in men, it may help explain why women generally exhibit 
greater pain sensitivity than men ${ }^{60}$ and are more likely to be diagnosed with most chronic pain disorders. ${ }^{61}$ Future research in chronic pain may explore new targets based on leptin pathways in humans. Leptin represents one of many physiological agents that can bridge multiple body systems, and may provide important clues on how to best manage chronic pain.

\section{Acknowledgments}

The WHI programs is funded by the National Heart, Lung, and Blood Institute, National Institutes of Health, U.S. Department of Health and Human Services through contracts, HHSN268201100046C, HHSN268201100001C, HHSN268201100002C, HHSN268201100003C, and HHSN268201100004C. The authors would like to acknowledge the WHI investigators: Program Office: (National Heart, Lung, and Blood Institute, Bethesda, Maryland) Dr. Jacques Rossouw, Shari Ludlam, Dr. Dale Bur-wen, Dr. Joan McGowan, Dr. Leslie Ford, and Dr. Nancy Geller; Clinical Coordinating Center: (Fred Hutchinson Cancer Research Center, Seattle, WA) Dr. Garnet Anderson, Dr. Ross Prentice, Dr. Andrea LaCroix, and Dr. Charles Kooperberg; Investigators and Academic Centers: (Brigham and Women's Hospital, Harvard Medical School, Boston, MA) Dr. JoAnn E. Manson; (MedStar Health Research Institute/ Howard University, Washington, DC) Dr. Barbara V. Howard; (Stanford Prevention Research Center, Stanford, CA) Dr. Marcia L. Stefanick; (The Ohio State University, Columbus, $\mathrm{OH}$ ) Dr. Rebecca Jackson; (University of Arizona, Tucson/Phoenix, AZ) Dr. Cynthia A. Thomson; (University at Buffalo, Buffalo, NY) Dr. Jean Wactawski-Wende; (University of Florida, Gainesville/Jacksonville, FL) Dr. Marian Limacher; (University of Iowa, Iowa City/Davenport, IA) Dr. Robert Wallace; (University of Pittsburgh, Pittsburgh, PA) Dr. Lewis Kuller; (Wake Forest University School of Medicine, Winston-Salem, NC) Dr. Sally Shumaker; Women's Health Initiative Memory Study: (Wake Forest University School of Medicine, Winston-Salem, NC) Dr. Sally Shumaker.

\section{Author Disclosure Statement}

No competing financial interests exist.

\section{References}

1. Faggioni R, Jones-Carson J, Reed DA, et al. Leptindeficient (ob/ob) mice are protected from $\mathrm{T}$ cell-mediated hepatotoxicity: Role of tumor necrosis factor alpha and IL18. Proc Natl Acad Sci USA 2000;97:2367-2372.

2. Shen J, Sakaida I, Uchida K, Terai S, Okita K. Leptin enhances TNF-alpha production via p38 and JNK MAPK in LPS-stimulated Kupffer cells. Life Sci 2005;77:15021515.

3. Canavan B, Salem RO, Schurgin S, et al. Effects of physiological leptin administration on markers of inflammation, platelet activation, and platelet aggregation during caloric deprivation. J Clin Endocrinol Metab 2005;90: 5779-5785.

4. Kutlu S, Canpolat S, Sandal S, Ozcan M, Sarsilmaz M, Kelestimur H. Effects of central and peripheral administration of leptin on pain threshold in rats and mice. Neuro Endocrinol Lett 2003;24:193-196.
5. Rodgers HM, Liban S, Wilson LM. Attenuated pain response of obese mice (B6.Cg-lep(ob)) is affected by aging and leptin but not sex. Physiol Behav 2014;123:80-85.

6. Tian Y, Wang S, Ma Y, Lim G, Kim H, Mao J. Leptin enhances NMDA-induced spinal excitation in rats: A functional link between adipocytokine and neuropathic pain. Pain 2011;152:1263-1271.

7. Gandhi R, Perruccio AV, Rizek R, Dessouki O, Evans HM, Mahomed NN. Obesity-related adipokines predict patientreported shoulder pain. Obes Facts 2013;6:536-541.

8. Lubbeke A, Finckh A, Puskas GJ, et al. Do synovial leptin levels correlate with pain in end stage arthritis? Int Orthop 2013;37:2071-2079.

9. Massengale M, Lu B, Pan JJ, Katz JN, Solomon DH. Adipokine hormones and hand osteoarthritis: Radiographic severity and pain. PLoS One 2012;7:e47860.

10. Perruccio AV, Mahomed NN, Chandran V, Gandhi R: Plasma adipokine levels and their association with overall burden of painful joints among individuals with hip and knee osteoarthritis. J Rheumatol 2014;41:334-337.

11. Fietta P, Fietta P. Counterbalance between leptin and cortisol may be associated with fibromyalgia. Psychiatry Clin Neurosci 2006;60:529-529.

12. Homann D, Louzada FM, Goes SM, et al. Acylated ghrelin: A potential marker for fibromyalgia? Eur J Pain 2013;17: 1216-1224.

13. Ablin JN, Aronov N, Shimon I, et al. Evaluation of leptin levels among fibromyalgia patients before and after three months of treatment, in comparison with healthy controls. Pain Res Manag 2012;17:89-92.

14. Stringer EA, Baker KS, Carroll IR, et al. Daily cytokine fluctuations, driven by leptin, are associated with fatigue severity in chronic fatigue syndrome: Evidence of inflammatory pathology. J Transl Med 2013;11:93.

15. Rusu C, Gee ME, Lagace C, Parlor M. Chronic fatigue syndrome and fibromyalgia in Canada: Prevalence and associations with six health status indicators. Health Promot Chronic Dis Prev Can 2015;35:3-11.

16. Dell'Osso L, Bazzichi L, Baroni S, et al. The inflammatory hypothesis of mood spectrum broadened to fibromyalgia and chronic fatigue syndrome. Clin Exp Rheumatol 2015;33: S109-S116.

17. Langer RD, White E, Lewis CE, Kotchen JM, Hendrix SL, Trevisan M. The Women's Health Initiative Observational Study: Baseline characteristics of participants and reliability of baseline measures. Ann Epidemiol 2003;13:S107-S121.

18. Liuzzi A, Savia G, Tagliaferri M, et al. Serum leptin concentration in moderate and severe obesity: Relationship with clinical, anthropometric and metabolic factors. Int $\mathbf{J}$ Obes Relat Metab Disord 1999;23:1066-1073.

19. McKendall MJ, Haier RJ. Pain sensitivity and obesity. Psychiatry Res 1983;8:119-125.

20. Ray L, Lipton RB, Zimmerman ME, Katz MJ, Derby CA. Mechanisms of association between obesity and chronic pain in the elderly. Pain 2011;152:53-59.

21. Rechardt M, Shiri R, Lindholm H, Karppinen J, ViikariJuntura E. Associations of metabolic factors and adipokines with pain in incipient upper extremity soft tissue disorders: A cross-sectional study. BMJ Open 2013;3:e003036.

22. Roane DS, Porter JR. Nociception and opioid-induced analgesia in lean $(\mathrm{Fa} /-)$ and obese (fa/fa) Zucker rats. Physiol Behav 1986;38:215-218.

23. Wilson AC, Samuelson B, Palermo TM. Obesity in children and adolescents with chronic pain: Associations 
with pain and activity limitations. Clin J Pain 2010;26: 705-711.

24. Wood D, Goodnight S, Haig AJ, Nasari T. Body mass index, but not blood pressure is related to the level of pain in persons with chronic pain. J Back Musculoskelet Rehabil 2011;24:111-115.

25. Okifuji A, Donaldson GW, Barck L, Fine PG. Relationship between fibromyalgia and obesity in pain, Function, mood, and sleep. J Pain 2010;11:1329-1337.

26. Ursini F, Naty S, Grembiale RD. Fibromyalgia and obesity: The hidden link. Rheumatol Int 2011;31:1403-1408.

27. Ho GY, Wang T, Gunter MJ, et al. Adipokines linking obesity with colorectal cancer risk in postmenopausal women. Cancer Res 2012;72:3029-3037.

28. Vadacca M, Margiotta DP, Navarini L, Afeltra A. Leptin in immuno-rheumatological diseases. Cell Mol Immunol 2011; 8:203-212.

29. Kim CK, Ryu WS, Choi IY, et al. Detrimental effects of leptin on intracerebral hemorrhage via the STAT3 signal pathway. J Cereb Blood Flow Metab 2013;33:944-953.

30. Jitprasertwong P, Jaedicke KM, Nile CJ, Preshaw PM, Taylor JJ. Leptin enhances the secretion of interleukin (IL)18 , but not IL-1beta, from human monocytes via activation of caspase-1. Cytokine 2014;65:222-230.

31. Maeda T, Kiguchi N, Kobayashi Y, Ikuta T, Ozaki M, Kishioka S. Leptin derived from adipocytes in injured peripheral nerves facilitates development of neuropathic pain via macrophage stimulation. Proc Natl Acad Sci USA 2009; 106:13076-13081.

32. Lafrance V, Inoue W, Kan B, Luheshi GN. Leptin modulates cell morphology and cytokine release in microglia. Brain Behav Immun 2010;24:358-365.

33. Tang CH, Lu DY, Yang RS, et al. Leptin-induced IL-6 production is mediated by leptin receptor, insulin receptor substrate-1, phosphatidylinositol 3-kinase, Akt, NF-kappaB, and p300 pathway in microglia. J Immunol 2007;179:12921302.

34. Ji RR, Berta T, Nedergaard M. Glia and pain: Is chronic pain a gliopathy? Pain 2013;154 Suppl 1:S10-S28.

35. Cristino L, Luongo L, Imperatore R, et al. Orexin-A and endocannabinoid activation of the descending antinociceptive pathway underlies altered pain perception in leptin signalling deficiency. Neuropsychopharmacology 2015;41:508-520.

36. Iannitti T, Graham A, Dolan S. Increased central and peripheral inflammation and inflammatory hyperalgesia in Zucker rat model of leptin receptor deficiency and genetic obesity. Exp Physiol 2012;97:1236-1245.

37. Iikuni N, Lam QL, Lu L, Matarese G, La Cava A. Leptin and inflammation. Curr Immunol Rev 2008;4:70-79.

38. Kwon H, Pessin JE. Adipokines mediate inflammation and insulin resistance. Front Endocrinol (Lausanne) 2013;4:71.

39. Milaneschi Y, Simonsick EM, Vogelzangs N, et al. Leptin, abdominal obesity, and onset of depression in older men and women. J Clin Psychiatry 2012;73:1205-1211.

40. Vuolteenaho K, Koskinen A, Moilanen E. Leptin-A link between obesity and osteoarthritis. Applications for prevention and treatment. Basic Clin Pharmacol Toxicol 2014; 114:103-108.

41. Spiegel K, Leproult R, L'Hermite-Baleriaux M, Copinschi G, Penev PD, Van Cauter E. Leptin levels are dependent on sleep duration: Relationships with sympathovagal balance, carbohydrate regulation, cortisol, and thyrotropin. J Clin Endocrinol Metab 2004;89:5762-5771.
42. Behnes M, Brueckmann M, Lang S, et al. Alterations of leptin in the course of inflammation and severe sepsis. BMC Infect Dis 2012;12:217.

43. Abbenhardt C, McTiernan A, Alfano CM, et al. Effects of individual and combined dietary weight loss and exercise interventions in postmenopausal women on adiponectin and leptin levels. J Internal medicine 2013;274: $163-175$.

44. Zaccaria M, Ermolao A, Brugin E, Bergamin M. Plasma leptin and energy expenditure during prolonged, moderate intensity, treadmill exercise. J Endocrinol Invest 2013;36: 396-401.

45. Relic B, Zeddou M, Desoroux A, Beguin Y, de Seny D, Malaise MG. Genistein induces adipogenesis but inhibits leptin induction in human synovial fibroblasts. Lab Invest 2009;89:811-822.

46. Singh UP, Singh NP, Guan H, et al. The emerging role of leptin antagonist as potential therapeutic option for inflammatory bowel disease. Int Rev Immunol 2014;33:23-33.

47. Guneli E, Gumustekin M, Ates M. Possible involvement of ghrelin on pain threshold in obesity. Med Hypotheses 2010; 74:452-454.

48. Lim G, Wang S, Zhang Y, Tian Y, Mao J. Spinal leptin contributes to the pathogenesis of neuropathic pain in rodents. J Clin Invest 2009;119:295-304.

49. Gandhi R, Santone D, Takahashi M, Dessouki O, Mahomed NN. Inflammatory predictors of ongoing pain 2 years following knee replacement surgery. Knee 2013;20: 316-318.

50. Saad MF, Riad-Gabriel MG, Khan A, et al. Diurnal and ultradian rhythmicity of plasma leptin: Effects of gender and adiposity. J Clin Endocrinol Metab 1998;83: 453-459.

51. Schoeller DA, Cella LK, Sinha MK, Caro JF. Entrainment of the diurnal rhythm of plasma leptin to meal timing. J Clin Invest 1997;100:1882-1887.

52. Li X, Kang L, Li G, et al. Intrathecal leptin inhibits expression of the $\mathrm{P} 2 \mathrm{X} 2 / 3$ receptors and alleviates neuropathic pain induced by chronic constriction sciatic nerve injury. Mol Pain 2013;9:65.

53. Fernandez-Martos CM, Gonzalez P, Rodriguez FJ. Acute leptin treatment enhances functional recovery after spinal cord injury. PLoS One 2012;7:e35594.

54. Yan BC, Choi JH, Yoo KY, et al. Leptin's neuroprotective action in experimental transient ischemic damage of the gerbil hippocampus is linked to altered leptin receptor immunoreactivity. J Neurol Sci 2011;303:100-108.

55. Olama SM, Elsaid TO, El-Arman M. Serum leptin in Egyptian patients with fibromyalgia syndrome: Relation to disease severity. Int J Rheum Dis 2013;16:583-589.

56. Alvarez P, Bogen O, Chen X, Giudice LC, Levine JD. Ectopic endometrium-derived leptin produces estrogendependent chronic pain in a rat model of endometriosis. Neuroscience 2014;258:111-120.

57. Ainslie DA, Morris MJ, Wittert G, Turnbull H, Proietto J, Thorburn AW. Estrogen deficiency causes central leptin insensitivity and increased hypothalamic neuropeptide Y. Int J Obes Relat Metab Disord 2001;25:1680-1688.

58. Pernambuco AP, Schetino LP, Alvim CC, et al. Increased levels of IL-17A in patients with fibromyalgia. Clin Exp Rheumatol 2013;31:S60-S63.

59. Zhang Z, Cherryholmes G, Mao A, et al. High plasma levels of MCP-1 and eotaxin provide evidence for an im- 
munological basis of fibromyalgia. Exp Biol Med (Maywood) 2008;233:1171-1180.

60. Mogil JS. Sex differences in pain and pain inhibition: Multiple explanations of a controversial phenomenon. Nat Rev Neurosci 2012;13:859-866.

61. Greenspan JD, Craft RM, LeResche L, et al. Studying sex and gender differences in pain and analgesia: A consensus report. Pain 2007;132 Suppl 1:S26-S45.
Address correspondence to: Jarred Younger, PhD

Department of Psychology University of Alabama at Birmingham 1300 University Boulevard Birmingham, AL 35233

E-mail: youngerlab@uab.edu 